\title{
NEWER DRUGS FOR VISCERAL LEISHMANIASIS: A REVIEW
}

\author{
${ }^{1}$ Krishna Pandey, ${ }^{2}$ Dharmendra Singh, ${ }^{3}$ Sanjiva Bimal, \\ ${ }^{1}$ Vidyanand Rabidas, ${ }^{4}$ Krishna Murti, ${ }^{5}$ Chadrashekhar Lal and ${ }^{2} \mathbf{P}$. Das \\ ${ }^{1}$ Department of Clinical Medicine, \\ ${ }^{2}$ Department of Molecular Biology, \\ ${ }^{3}$ Department of Immunology, \\ ${ }^{4}$ Department of Biochemistry, \\ Rajendra Memorial Research Institute of Medical Sciences, \\ Indian Council of Medical Research, Agamkuan Patna 800007, Bihar, India \\ ${ }^{5}$ Department of Pharmacy Practice, National Institute of Pharmaceutical Education and Research, Hajipur, Bihar, India
}

Received 2014-03-26; Revised 2014-05-01; Accepted 2014-05-05

\begin{abstract}
Visceral Leishmaniasis (VL) is one of the most neglected tropical diseases worldwide. The diagnosis and treatment of this disease is quite complex. Sodium Antimony Gluconate (SAG) which used to be a very effective treatment has now developed resistance and is potentially a cardio-toxic drug. Pentamidine has now been discarded because of adverse effect of diabetes mellitus. Amphotericin-B is an effective drug but can cause nephrotoxicity. Miltefosine is a new oral effective drug which has recently been introduced in the kala-azar elimination program in the Indian subcontinent. Paromomycin is an injectable aminoglycoside which is quite cheap but can cause ototoxicity and nephrotoxicity. Sitamaquine, an oral antimalarial drug is still in phase-II trial stage. Combination Therapy is been tried with good results. Single dose Ambisome (liposomal Amphotericin-B) though costly is a very good alternative.
\end{abstract}

Keywords: Visceral Leishmaniasis, Miltefosine, Paromomycin, Sitamaquine, Combination Therapy, Ambisome

\section{INTRODUCTION}

Visceral Leishmaniasis (VL) is a disease of major public health importance in India, particularly Bihar. As is well known, it is caused by a protozoan parasite, Leishmania donovani and transmitted by the bite of female sand fly vector, Phlebotomus argentipes. The worldwide prevalence and incidence of VL are 2.5 million and 0.5 million respectively per year and about 350 million people are at risk whereas 500000 people suffer from the disease (Bhattacharya et al., 2006). About $90 \%$ of the cases occur in five countries namely India, Nepal, Sudan, Bangladesh and Brazil. In India, about 165 million people are at risk with 20000 new cases (Bora, 1999), out of which about $90 \%$ occur in Bihar state alone apart from other states like Uttar Pradesh, West Bengal and Jharkhand. The disease affects the poorest of the poor and about $90 \%$ of the people affected with VL earn less than $2 \$$ a day (Sinha et al., 2005). The disease is regarded as one of the most neglected diseases.

The diagnostic and management aspect of the disease is quite difficult. Management involves various issues like (a) fewer number of drugs (b) resistance to drugs like Sodium Antimony Gluconate (SAG) (c) mostly injectable and toxic drugs (d) costly drugs (e) increasing proportion of Leishmania-HIV co-infection and finally (f) lack of an effective vaccine. With the increasing unresponsiveness to drugs like SAG (which has come down to as low as $40 \%$ in Bihar) various clinical drug trials on VL/Kala-azar are being undertaken (Das et al., 2005).

\subsection{Clinical Drug Trials}

Miltefosine or Hexadecylphosphocholine, is an antineoplastic agent previously used as a topical logy, Rajendra Memorial Research Institute of Medical Sciences, Agamkuan, Patna-800 007, Bihar, India 
application for the treatment of skin metastasis of breast cancer. It was shown to have good action against Leishmania donovani in vitro. The exact mechanism of action is not well known. However, it has been shown to produce apoptosis and block signaling pathways.

In the phase III study, 80 children in the age group of 2 to 11 years were included in the study according to inclusion and exclusion criteria. This trial was conducted at four Kala-azar centers of excellence in Bihar including this Institute. The study revealed $94 \%$ final cure rate when used in the in the dose of $2.5 \mathrm{mg} \mathrm{kg}^{-1}$ body wt. for 28 days after meals orally with few gastrointestinal toxic effects like diarrhoea and vomiting, raised serum Alanine Transaminase (ALT) and Aspartate Transaminase (AST) levels in the second and third week which came down to normal at the end of treatment. A few patients (about 1 to $2 \%$ ) had a marginal increase in Blood Urea Nitrogen (BUN) and serum creatinine levels. Both these adverse events did not require treatment discontinuation (Bhattacharya et al., 2004).

The Phase IV study of Miltefosine was conducted in children and adults over 13 centers in Bihar where this Institute was one of the clinical centre as well as the coordinating centre. Miltefosine was given in the same dose as in Phase III study according to inclusion and exclusion criteria (2 to 65 years) under Outdoor Patient Setting (OPD). In adults weighing $>25 \mathrm{~kg}$, a total dose of $100 \mathrm{mg}$ per day was given in two divided doses after meals. Altogether about 1500 patients were enrolled and the results and adverse events were a replica of the Phase III study (final cure rate of about 94\%). Relapse patients were treated with intravenous Amphotericin B injection (Bhattacharya et al., 2007). Three female patients became pregnant during the follow up period and these were followed up to the birth of the fetus for possible teratogenic side effects. All the three had full term normal pregnancy with no evidence of any teratogenicity. However, since the details in pregnant women are not fully known and it had not been tried on a large scale in such women, the use of Miltefosine is not advocated in pregnancy.

Miltefosine is one of the few drugs, which even though being an anti-neoplastic agent, stimulates the bone-marrow rather than suppress it like other anticancer drugs. This in turn leads to correction of pancytopenia including hemoglobin, which is a regular feature of VL. Miltefosine, being an oral agent, can be easily given at an outpatient basis. This drug has been recommended by the WHO in the Kala-azar elimination program in India, Nepal and Bangladesh. The main limiting factor of this drug is its long half-life of about 5 days, which can lead to development of early resistance besides its high cost. At present World Bank sponsored Pharmacovigilance program is being done with Miltefosine in few selected endemic districts of Bihar.

After the launching of Miltefosine, a new aminoglycoside drug namely Paromomycin was tried in VL under sponsorship of Institute of One World Health (iOWH), USA. The Phase III study was undertaken to study the safety and efficacy of Paromomycin and to compare it with the standard control reference arm i.e., injectable Amphotericin B. Paromomycin was given in the dose of $15 \mathrm{mg}$ per kg body wt. deep intramuscular for 21 days after fulfilling the inclusion and exclusion criteria. The age group was 5 to 55 years and both sexes were chosen for the study. Altogether 667 patients were included at four centers in Bihar. These patients were randomized in a 3:1 ratio (Paromomycin: Amphotericin B i.e., 500 Paromomycin Vs. 167 Amphotericin B). The main toxic effects were a transient rise in serum creatinine and BUN levels (nephrotoxicity) as well as reversible ototoxicity, which was assessed by audiometry. The most common Adverse Event (AE) observed was pain at injection site. The final cure rate was about 95\% (Sundar et al., 2007). However, being an aminoglycoside drug this drug has an advantage of being cheap. The Phase IV trials on Paromomycin, has just been completed. This is a very cheap drug but being an aminoglycoside is more prone to develop resistance. Hence it is being tried in combination studies.

Another drug Sitamaquine, an 8-aminoquinoline (anti-malarial) has been tried for VL (Sundar and Chaterjee, 2006). In the Phase II study, sponsored by GSK (Glaxo-Smithkline Beecham), 48 patients were included. The control arm was injectable Amphotericin B. Sitamaquine was given in the dose of $2 \mathrm{mg}$ per $\mathrm{kg}$ body wt. for 21 days orally. The study revealed initial cure rate of $95 \%$ and the final cure rate of $85 \%$. This drug has to be monitored for nephrotoxicity as it can cause acute glomurelonephritis. It is not to be given in patients with glucose-6-phosphate dehydrogenase deficiency as it can cause severe haemolysis. The drug can also cause cyanosis due to increase in the level of methemoglobinaemia. Sitamaquine is still in a primitive trial stage and further trials are to be assessed before advocacy of this drug for the treatment of VL.

In view of minimizing the dose and duration of the existing anti-VL drug, the combination trial to compare the safety and efficacy of various combination like liposomal Amphotericin B (AmBisome) with 
Miltefosine, Paromomycin with Miltefosine and AmBisome with Paromomycin. These are basically being tried for a shorter duration of treatment of VL, but can also prove to be a boon for treating patients with HIV-VL co-infection (Pandey et al., 2005). The phaseIII trials sponsored by DNDi were conducted with a combination of Miltefosine and Paromomycin showing 98.7\% cure rate, Ambisome + Miltefosine and Ambisome + Paromomycin having a cure rate of $97.5 \%$ whereas single dose Ambisome $\left(10 \mathrm{mg} \mathrm{kg}^{-1}\right)$ had a cure rate of $96 \%$. These trials are still in a very early stage and the final outcome will be known after a few more years (Sundar et al., 2011). The phase-IV trials sponsored by DNDi are being conducted in a few endemic districts of Bihar. The combination therapies can be used as a drug for the future as they are very less prone for resistance. As per the latest suggestion single dose Ambisome could be used for complicated cases like HIV-VL and VL-Tuberculosis co-infection.

\section{CONCLUSION}

All-in-all, Amphotericin B, Paromomycin and Miltefosine appear to be good drugs for VL with a very high cure rate and few side effects. The first two are injectable drugs whereas the other one is an oral preparation. Amphotericin B requires hospitalization and electrolyte monitoring, which is not the case with Miltefosine as it can be given on an outpatient basis. The future of the other drug Sitamaquine is still not very clear and Paromomycin is in the Phase IV stage. The future of combination treatment is still not very clear. Single dose Ambisome could prove to be a future and effective short course alternative for treatment with minimal side effects (Sundar et al., 2010). Further keeping in view the rising trend of unresponsiveness to SAG (Das et al., 2005) and the increasing incidence of HIV-VL co-infection, which is very prone to relapses as well as lack of an effective vaccine for VL warrants future drug trials.

\subsection{Conflicts of Interest}

The researcher declare that they have no conflicts of interest.

\section{ACKNOWLEDGEMENT}

The researcher wish to thank the sincere efforts of Mr. Dhirendra Kumar, CRC and Mr. Naresh Kumar Sinha, for the sincere efforts rendered by them in the preparation of manuscript.

\section{REFERENCES}

Bhattacharya, S.K., P.K. Sinha, S. Sundar, C.P. Thakur and T.K. Jha et al., 2007. Phase 4 trial of miltefosine for the treatment of Indian visceral Leishmaniasis. J. Infect. Dis., 196: 591-8. DOI: 10.1086/519690

Bhattacharya, S.K., S. Dipika and J. Karbwang, 2006. Childhood visceral leishmaniasis. Indian J. Med. Res., 123: 353-356. PMID: 16778316

Bhattacharya, S.K., T.K. Jha, S. Sundar, C.P. Thakur and J. Engel et al., 2004. Efficacy and tolerability of miltefosine for childhood visceral Leishmaniasis in India. Clin. Infec. Dis., 38: 217-221. DOI: $10.1086 / 380638$

Bora, D., 1999. Epidemiology of Leishmaniasis in India. Nat. Med. J. Ind., 12: 62-68.

Das, V.N.R., A. Ranjan, V.P. Singh, N.A. Siddiqui and P.K. Sinha et al., 2005. Magnitude of unresponsiveness Sodium stibogluconate for the treatment of Visceral Leishmaniasis in Bihar. Nat Med. J. Ind., 18: 131-33.

Pandey, K., P.K. Sinha, V.N.R. Das, N. Kumar and S.M. Hasan et al., 2005. HIV-1 infection, visceral leishmaniasis, Koch's chest and tuberculous meningitis in the same patient-A case report. Ann Trop Med. Parasitol., 99: 807-11. DOI: 10.1179/136485905X75386

Sinha, P.K., K. Pandey and S.K. Bhattacharya, 2005. Diagnosis and management of Leishmania/HIV coinfection. Indian J. Med Res., 121: 407-414.

Sundar, S. and M. Chaterjee, 2006. Visceral Leishmaniasis-current therapeutic modalities. Indian J. Med. Res., 123: 345-352.

Sundar, S., J. Chakravarty, D. Agarwal, M. Rai and H.W. Murray, 2010. Single-dose liposomal Amphotericin B for visceral leishmaniasis in India. N Engl. J. Med., 11: 504-512. DOI: 10.1056/ NEJM oa 0903627

Sundar, S., P.K. Sinha, M. Rai, D.K. Verma and K. Nawin et al., 2011. Comparison of short-course multidrug treatment with standard therapy for visceral leishmaniasis in India: An open-label, noninferiority, randomised controlled trial. Lancet, 377: 477-486. DOI: 10.1016/S0140-6736(10)62050-8

Sundar, S., T.K. Jha, C.P. Thakur, P.K. Sinha and S.K. Bhattachaya, 2007. Injectable paromomycin for Visceral Leishmaniasis in India. New England J. Med., 356: 71-81. DOI: 10.1056/NEJMoa066536 